\title{
Antifungal Activity of Crude Glycolated Extracts of Solanum tuberosum L. (White Potato) Peelings against Candida and Aspergillus Species
}

\author{
Jaime O. Yu, ${ }^{1}$ Albert L. Yap, ${ }^{1}$ Alexandria A. Tuason, ${ }^{1}$ Cyrene C. Tan, ${ }^{1}$ Hansley T. Tan, ${ }^{1}$ Leonard V. Tan, ${ }^{1}$ \\ Nicole G. Tan, ${ }^{1}$ Ria T. Tan, ${ }^{1}$ Denzy I. Tangkusan, ${ }^{1}$ Jon S. Tiosin, ${ }^{1}$ Ivi C. Torres, ${ }^{1}$ Jayson R. Trinchera, ${ }^{1}$ \\ Cherry F. Tumampo, ${ }^{1}$ Jeanie K. Uy, ${ }^{1}$ Mary R. Uy, ${ }^{1}$ Francesco T. Valdecañas, ${ }^{1}$ Emmanuel M. Velasco, Jr., ${ }^{1}$ \\ Jesus B. Villeza, Jr., ${ }^{1}$ Jasper R. Viloria, ${ }^{1}$ Celina D. Yap, ${ }^{1}$ Jose T. Reyes ${ }^{2,3}$ and Irma R. Makalinao ${ }^{2}$ \\ ${ }^{1}$ College of Medicine, University of the Philippines Manila \\ ${ }^{2}$ Department of Pharmacology and Toxicology, College of Medicine, University of the Philippines Manila \\ ${ }^{3}$ Department of Neurosciences, College of Medicine and Philippine General Hospital, University of the Philippines Manila
}

\begin{abstract}
Background. A rise in the number of immunocompromised patients has increased the risk of opportunistic fungal infections. Identifying novel sources of antifungal agents from commonly discarded materials (i.e. potato peelings) can provide a cheaper alternative for antifungal drugs.
\end{abstract}

Objectives. The aim of the study was to determine the antifungal activity of crude glycoalkaloid extract from Solanum tuberosum L. (white potato) peelings against opportunistic fungi Candida albicans, Candida glabrata, Aspergillus niger, Aspergillus fumigatus, and Aspergillus flavus.

Methods. The glycoalkaloid content from dried potato peelings were extracted using ethanol and confirmed using colorimetric tests.The extract had a concentration 833.33 microgram $/ \mathrm{mL}$. Determination of the minimum inhibitory concentrations (MICs) of the extract via two-fold broth dilution was performed for the five fungi with amphotericin $B$ and fluconazole as the reference antifungal drugs.

Results. MICs of the crude extract for C. albicans, C. glabrata, A. fumigatus, and A. niger were not found within the concentration range of the studies and would thus need further experiments using a broader range of glycoalkaloid concentrations. The extract was found to have a MIC of 104.17 microgram $/ \mathrm{mL}$ for $A$. flavus, thereby verifying the antifungal effect of glycoalkaloid against $A$. flavus at said concentration.

Conclusion. Glycoalkaloids from Solanum tuberosum are a potential source of antifungals against certain opportunistic fungi.

Key Words: Antifungal agent, opportunistic fungal infections, Solanum tuberosum, potato, glycoalkaloids, Candida, Aspergillus

\section{INTRODUCTION}

Presented and awarded "Best Paper" at the 18th Anniversary of the National Institutes of Health, 14th UP Manila Science and Technology Week, 7th Anniversary of the Metro Manila Health Research and Development Consortium on February 17-18, 2016.

Corresponding author: Jaime O. Yu

College of Medicine

University of the Philippines Manila

547 Pedro Gil St., Ermita, Manila 1000, Philippines

Telephone: +6325168032

Email: jaime_antonio_yu@yahoo.com
Healthcare-acquired infections (HAI) are common threats to patients resulting in increased morbidity and mortality. An increasing trend in morbidity has been presented by the World Health Organization across countries in Southeast Asia, Eastern Mediterranean, Europe, and Western Pacific from the years 2002 to 2011, and is continuing to rise worldwide due to a plurality of factors. ${ }^{1}$ Two of the most common fungi causing HAIs are Candida and Aspergillus species. In 2016, a total of 1,852,137 severe fungal infections were estimated to have occurred in the 
Philippines, with Aspergillus and Candida accounting for $10.9 \%$ and $80.4 \%$ of the total cases, respectively. ${ }^{2}$

A potential source of new antimicrobial is the commonly marketed crop, Solanum tuberosum (white potato) (Figure 1). Plants belonging to the family Solanaceae have been of great interest for several of their medicinal properties. ${ }^{3}$ Solanum tuberosum is found to contain glycoalkaloids which are toxic secondary metabolites produced during stress, such as during pest or fungal attack, for the protection of the crop. ${ }^{4}$ These metabolites also contribute to the bitter taste and burning irritation to the tongue when eaten.
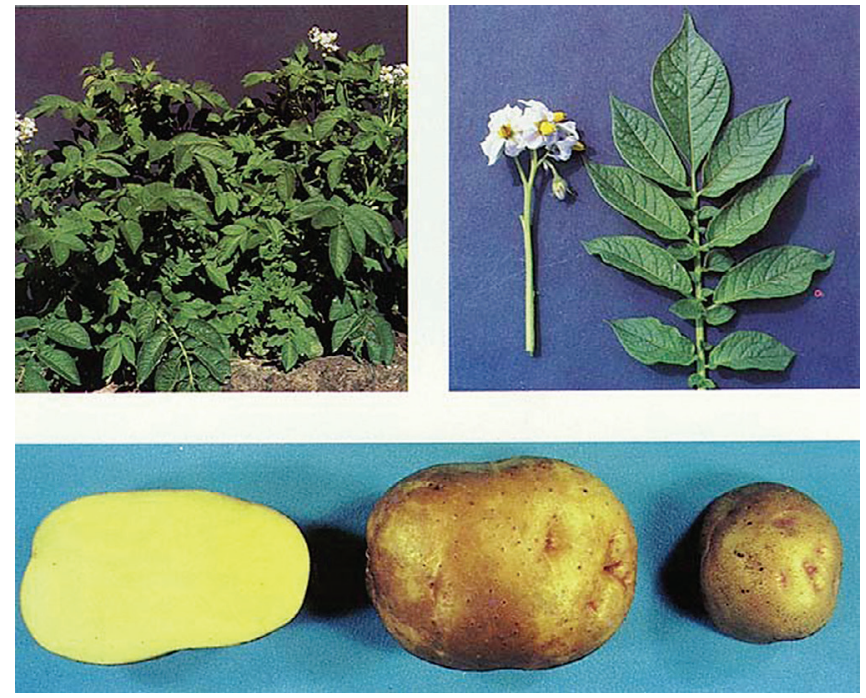

Figure 1. Solanum tuberosum (white potato). Photo taken from Potato Association of America (PotatoAssociation.org).

Due to the rise in number of immunocompromised individuals in recent years, the proliferation of opportunistic microorganisms has been more of a global concern. Primarily, this study addressed the need for a novel source of antifungal agent to combat common fungal species affecting immunocompromised individuals in healthcare facilities. It aimed to authenticate the effect of Solanum tuberosum glycoalkaloid extracts on the growth of the common opportunistic fungal microbes Candida and Aspergillus. In so doing, it also strove to come up with an antifungal agent from readily available sources and their commonly discarded parts. This is also in light of finding a cheaper alternative for the costly medications given to patients with fungal infections. It is an advantage that the particular species of potato being studied is highly available, accessible, and locally produced. Thus, acquisition of the raw materials for experimentation is therefore straightforward.

\section{MATERIALS AND METHODS}

\section{Collection of Materials}

Potatoes used for the experiment were sourced from a local farm that grows non-GMO (genetically modified organisms) potatoes organically. It was ensured that all the potatoes used were sourced from the same farm and certified as Solanum tuberosum L. by the Philippine Department of Agriculture Bureau of Plant Industry.

\section{Glycoalkaloid Extraction}

The Solanum tuberosum L. tubers were mechanically separated from their peels via abrasion method after thorough washing with $10 \%$ sodium bicarbonate. The peels were then subjected to air drying for four weeks before maceration by a blender. The peel fragments were then stored in clean, airtight bottles away from sunlight.

The extraction method employed was adapted and modified from that of Bushway, Bureau, and Stickney using ethanol as the extracting solvent. ${ }^{5}$ Finely macerated peels weighing $75 \mathrm{~g}$ were soaked with $300 \mathrm{~mL}$ of analytical reagent-grade ethanol for 72 hours at room temperature away from sunlight. The mixture was then filtered and subjected to rotary evaporation until the volume had reached $25 \mathrm{~mL}$. Afterwards, $2 \mathrm{~mL}$ of glacial acetic acid was added to the solution. After centrifugation at $38000 \mathrm{~g}$ for 10 minutes, $25 \mathrm{~mL}$ of concentrated ammonium hydroxide was added to precipitate the glycoalkaloids. The samples were then divided into separate tubes and subjected to water bath at $70^{\circ} \mathrm{C}$ for 30 minutes. These tubes were then refrigerated overnight.

After refrigeration, the tubes were again subjected to centrifugation at $38000 \mathrm{~g}$ for 10 minutes. Pellets were pooled into a single container and sent for lyophilization. After drying, the extract was weighed before being stored in a freezer at $-5^{\circ} \mathrm{C}$.

\section{Confirmatory Tests for the Presence of Glycoalkaloids}

The extract was redissolved in dimethyl sulfoxide (DMSO) and was subjected to confirmatory tests to confirm the presence of alkaloids. The reagents used were the following: Mayer's reagent (potassium mercuric iodide solution), Wagner's reagent (iodine solution in potassium iodide), Hager's reagent (picric acid saturated solution), and Valser's reagent (mercuric iodide test solution). One milliliter $(1 \mathrm{~mL})$ of each reagent was added to $0.5 \mathrm{~mL}$ of the solution to check for the formation of colored precipitate that would verify the presence of alkaloid functional groups in the extract.

\section{Determination of Minimum Inhibitory Concentration (MIC)}

The minimum inhibitory concentration was investigated via two-fold broth dilution in accordance with the European Committee on Antimicrobial Susceptibility Testing (EUCAST) documents entitled, "Method for the determination of broth dilution minimum inhibitory concentration of antifungal agents for yeasts" and "Method for the determination of broth dilution minimum inhibitory concentration of antifungal agents for conidia- 
forming moulds." ${ }^{16-7}$ These methods used fluconazole and amphotericin B as reference drugs for the various Candida and Aspergillus species.

Stock solutions containing fluconazole and amphotericin $\mathrm{B}$ were made taking into consideration the potency of the powdered drug using DMSO as the solvent for both drugs. The stock solutions contained 25,600 microgram $/ \mathrm{mL}$ and 3,200 microgram $/ \mathrm{mL}$ for fluconazole and amphotericin B, respectively. The crude extract had a stock concentration of $166.67 \mathrm{mg} / \mathrm{mL}$.

Into the tubes designated as the tubes of largest concentration containing $3.96 \mathrm{~mL}$ of modified RPMI-1640 broth with $2 \%$ glucose and 3-(N-morpholino)propanesulfonic acid (MOPS) buffer, 40 microliters of the stock solutions were added to form $4 \mathrm{~mL}$, yielding a 100 -fold dilution of the stock solutions. After mixing, 2-fold serial dilution was carried out by obtaining $2 \mathrm{~mL}$ from the previous tube and adding these into new tubes containing $2 \mathrm{~mL}$ of the same modified RPMI1640 medium. This step was repeated until the last tube had been reached and the final $2 \mathrm{~mL}$ volume disposed of.

For Candida species, fungi were directly transferred aseptically using an inoculating loop to sterile distilled water. For Aspergillus species, conidia were first dislodged using a wash of polysorbate 20 (Tween ${ }^{\circledR} 20$ ) and sterile distilled water along with gentle scraping of the fungi. The fungal suspensions were added to sterile distilled water until turbidity had reached $0.5 \mathrm{McF}$ arland standard, and was then diluted ten-fold to reach a concentration of $1 \times 10^{5}$ colony forming units (CFU). A volume of $2 \mathrm{~mL}$ was used as inoculum in each tube to reach the desired concentrations of the standard drugs and the extract.

After addition of inoculum, the final concentrations of the extract were from $833.33 \mathrm{microgram} / \mathrm{mL}$ to $1.63 \mathrm{microgram} /$ $\mathrm{mL}$. Final concentrations for the reference antifungals ranged from 128 microgram $/ \mathrm{mL}$ to 0.25 microgram $/ \mathrm{mL}$ for fluconazole and 16 microgram $/ \mathrm{mL}$ to 0.0625 microgram $/ \mathrm{mL}$ for amphotericin B.

Each organism had setups performed in triplicate. Tubes containing Candida were incubated for 24 hours while tubes containing Aspergillus were incubated for 48 hours.

Three control tubes were used in every run for each organism and were designated as follows: negative control (broth and fungal inoculum), positive control (broth and sterile water), and blank control (broth and crude extract).

The minimum inhibitory concentration was determined to be the smallest concentration wherein growth had been inhibited as determined visually using a turbidity comparison card.

\section{Data Collection and Analysis}

The minimum inhibitory concentrations were recorded for each trial. Results for Aspergillus spp. and Candida spp. for fluconazole and amphotericin B were compared to those of the breakpoints listed by the EUCAST for the specific organisms.

\section{RESULTS}

\section{Confirmatory Tests}

All of the qualitative tests verified the presence of alkaloid functional groups in the extract through the production of precipitates after mixing (Table 1).

Table 1. Qualitative test results for the presence of alkaloids in the extract

\begin{tabular}{ccc} 
Reagent & Presence of Precipitates & Color of Precipitate \\
Mayer's & + & White \\
Wagner's & + & Brick-red \\
Valser's & + & Creamy white \\
Hager's & + & Yellow \\
\hline
\end{tabular}

\section{Determination of Minimum Inhibitory Concentration}

Minimum inhibitory concentrations (MICs) for the reference drugs showed that none of the organisms tested were resistant strains according to EUCAST breakpoints although $A$. fumigates was found to have intermediate susceptibility to amphotericin B (Table 2). The results of A. fumigatus were taken by the authors as still valid for use against the glycoalkaloid extract as the strain was not resistant to Amphotericin B.

Only A. Alavus was inhibited by the extract through MIC determination (Table 3). In all other trials, each organism was able to grow in the tube of highest concentration; thus, their respective MICs were outside the range of the MIC concentrations tested for the extract.

Table 2. EUCAST reference antifungal mic breakpoints (microgram/ml) and actual results for Fluconazole and Amphotericin B. ${ }^{28-30}$

\begin{tabular}{|c|c|c|c|c|c|c|}
\hline \multirow{3}{*}{ Fungus } & \multicolumn{6}{|c|}{ MIC Breakpoints (microgram/mL) } \\
\hline & \multicolumn{2}{|c|}{ Fluconazole (Control) } & \multicolumn{2}{|c|}{ Amphotericin B (Control) } & \multirow{2}{*}{$\begin{array}{l}\text { Result of Study Organisms } \\
\text { to Fluconazole or } \\
\text { Amphotericin B }\end{array}$} & \multirow{2}{*}{$\begin{array}{l}\text { Susceptibility of } \\
\text { Study Organisms to } \\
\text { Control Antifungals }\end{array}$} \\
\hline & Susceptible & Resistant & Susceptible & Resistant & & \\
\hline C. albicans & $\leq 2$ & $>4$ & -- & -- & 2 & Susceptible to Fluconazole \\
\hline C. glabrata & -- & -- & $\leq 1$ & $>1$ & 0.0625 & Susceptible to Amphotericin B \\
\hline A. fumigatus & -- & -- & $\leq 1$ & $>2$ & 2 & $\begin{array}{l}\text { Intermediate Susceptibility } \\
\text { to Amphotericin } \mathrm{B}^{*}\end{array}$ \\
\hline A. flavus & -- & -- & $\begin{array}{l}\text { (The MIC brea } \\
\text { than those } \mathrm{P}\end{array}$ & $\begin{array}{l}\text { its are higher } \\
\text { fumigatus.) }\end{array}$ & 1 & Susceptible to Amphotericin B \\
\hline A. niger & -- & -- & $<1$ & $>2$ & 0.5 & Susceptible to Amphotericin B \\
\hline
\end{tabular}


Table 3. Measured minimum inhibitory concentrations of extract and reference antifungals for Aspergillus and Candida species tested (microgram $/ \mathrm{ml}$ ) and EUCAST breakpoints for the respective antifungals used

\begin{tabular}{cc} 
Fungus & Extract \\
A. flavus & Inhibited at $104.17(2 / 3 \text { trials })^{*}$ \\
A. fumigatus & $>833.33(3 / 3$ trials $)$ \\
A. niger & $>833.33(3 / 3$ trials $)$ \\
C. albicans & $>833.33(3 / 3$ trials $)$ \\
C. glabrata & $>833.33(3 / 3$ trials $)$ \\
\hline
\end{tabular}

*Note: One trial had a MIC of $>833.33$ microgram $/ \mathrm{mL}$.

\section{DISCUSSION}

Candida are human pathogenic yeasts which are ubiquitous in the environment, with some species belonging to the normal human microbiota. ${ }^{8-10}$ These Candida species are among the most common causes of fungal infections worldwide, representing nearly $96 \%$ of all opportunistic mycoses. ${ }^{11-13}$ In the Philippine setting, candidiasis caused by Candida albicans ranks first among the common opportunistic infections due to the hot and humid weather coupled with the overgrowth of the diploid fungus. ${ }^{14}$ Candida glabrata, on the other hand, ranks second to third among other species in causing systemic or superficial candidal infection. ${ }^{15-16}$

Aspergillus is a mold commonly found both indoors (i.e. beddings), and outdoors (i.e. decomposing plant materials). It produces spores that are easily aerosolized, and causes infection when inhaled by susceptible hosts during extended antibiotic treatment and in instances of severe immunosuppression. Aspergillus fumigatus is now considered as the most prevalent airborne fungal pathogen with an increasing number of cases of fatal invasive aspergillosis in the immunocompromised. ${ }^{17}$ Aspergillusflavus is the second leading cause of human invasive aspergillosis and is the most common cause of superficial infection. ${ }^{18}$ Aspergillus niger, in contrast, is rarely reported as a causative agent of infection but is correlated with a $75 \%$ mortality rate for those infected. ${ }^{19}$

Extracts of Solanum plants have been proven effective in inducing antimicrobial effect against Staphylococcus aureus has been found to be significantly inhibited by the glycoalkaloid content of Solanum nigrum at a concentration of 20 microgram $/ \mathrm{mL} .{ }^{20-22}$

Extracted solanine from Solanum lycocarpum was shown to be inhibitory to the dermatopyteTrichophyton rubrum at a concentration of $25 \mathrm{microgram} / \mathrm{mL} .{ }^{23}$

The amount of glycoalkaloids in potato peels is closely associated with the production of chlorophyll, which causes the visible greening on the tuber surface. These processes are independent of each other but are both activated by light. Glycoalkaloid concentrations in the tuber decrease from the epidermis inwards with the highest amounts being found within the periderm and outer parenchymal tissue while having negligible concentrations in the pith. ${ }^{24} \mathrm{~A}$ level of 20 $\mathrm{mg}$ of glycoalkaloid is reported to be toxic to humans. ${ }^{25}$
Analysis of the specific glycoalkaloids reveals that $\alpha$-chaconine and $\alpha$-solanine are the two major glycoalkaloids found in commercial potatoes - comprising about $95 \%$ of the total glycoalkaloids in potato tubers. ${ }^{4}$ Solanine and chaconine have both fungicidal and pesticidal properties, having a synergistic reaction causing liposome lysis. ${ }^{24}$

A study by Zhao et al. looked into the antifungal activity of five solanaceous glycoalkaloids against Cercosporella brassicae and Alternaria porri, two phytopathogenic fungi. It was shown that a mixture of $\alpha$-solanine and $\alpha$-chaconine resulted in a marked synergistic antifungal activity. The combination of both glycoalkaloids produced a higher activity at lower concentrations than at higher concentrations. ${ }^{27}$

Another study by Fewell and Roddick in 1997 found that $\alpha$-chaconine was more inhibitory than $\alpha$-solanine on spore germination and on growth in liquid culture of Alternaria brassicicola and Phoma medicaginis, as well as on the growth of Ascobolus crenulatus and Rhizoctonia solani. At 50 micromoles or less, solanine caused little $(<20 \%)$ or no inhibition; but in combination with comparable concentrations of chaconine (which were sometimes also below the activity threshold), synergistic inhibitory effects were apparent by up to $100 \%{ }^{27}$

Generally, the effect of crude glycoalkaloid extract on A. flavus may be attributed to the surfactant effects on the fungi's sterol-containing cell membranes. Glycoalkaloid compounds destabilize the membrane forming complexes between the glycoside and membrane bound sterols such as those found in fungal hyphae. ${ }^{23}$

The study was able to successfully extract glycoalkaloids from Solanum tuberosum peels with ethanol as the extracting solvent as shown by the presence of colored precipitates using the confirmatory reagents. The antifungal activity of these ethanolic glycoalkaloid-containing extracts of $S$. tuberosum presented with inhibition of only Aspergillus flavus among the five organisms tested. This fungus had an MIC of 104.17 microgram $/ \mathrm{mL}$ while the MICs for C. albicans, C. glabrata, $A$. fumigatus, and $A$. niger were outside the concentration range of the crude extract used in the experiment. It is thus highly possible that certain interspecies variations in cell wall composition make $A$. flavus more susceptible to the action of the extract than any of the other fungi tested. However, it is noted that the antifungal activity of Solanum tuberosum in these fungal species cannot be completely ruled out even though the MICs were not determined in the experiment.

The use of reference antifungal drugs was not meant to be a means of comparison of activity. Rather, amphotericin B and fluconazole were used in order to ensure that the fungi used in the experiment were not resistant strains that would otherwise lead to falsely negative results. Standardization was ensured through the usage of the same broth, inoculum size, solvent concentration (less than 1\% DMSO), incubation time, and temperature throughout the experiment. The MICs for the respective drugs tested on each fungal species fell within the susceptible range, with the exception of $A$. fumigatus which had intermediate susceptibility. As stated 
in the EUCAST breakpoints for Candida and Aspergillus species, it is possible to conclude that the fungal species used were not resistant strains and would less likely provide falsely negative results in MIC determination.

Controls in the experiment were used in order to increase the validity of the results. A negative control was used in order to see how the growth of the specific fungus would appear, a positive control was used to see the appearance of the tubes that do not exhibit growth, and because the extract showed coarse flocculation that settled in the bottom of the tube, a blank control was used in order to avoid falsely reporting fungal growth. Also, the broth in the blank control did not become turbid and the color did not change from red to yellow which would have otherwise indicated growth of carbohydrate-fermenting organisms. In determining the MIC, it was ensured that the tubes were compared to these controls.

Possible reasons for the lack of positive results against the other fungi tested may be attributed to the inadequate concentration of extract applied during the assay to inhibit their growth. It is desired that nothing other than the glycoalkaloids and the drugs would be the sole producers of antifungal property. However, being an unpurified substance, the extract may have had unaccounted substances that may have affected the results.

\section{CONCLUSION}

The experiment showed that the crude glycoalkaloid extract from $S$. tuberosum peels has antifungal activity to a certain extent. The MIC of the extract for A. flavus was determined to be 104.17 microgram $/ \mathrm{mL}$. However, the experiment failed to demonstrate the antifungal activity within the concentrations tested for $A$. fumigatus, $A$. niger, $C$. albicans, and C. glabrata; hence, MICs for these fungi species were not determined. It should be noted that even though the MICs were not obtained, it does not necessitate that antifungal activity is absent. Further investigation is recommended to test antifungal activity at broader concentrations.

\section{RECOMMENDATION}

Several suggestions to further the study are recommended by its proponents. First, purification of the extract via absorption spectrophotometry, chromatography, or other quantitative confirmatory techniques is proposed in order to obtain a pure glycoalkaloid extract to further establish a stronger basis for the antifungal activity of glycoalkaloids.

Second, studies that use a broader range of concentrations of extracts are recommended in order to ascertain the MICs of the extract for the other organisms. Further studies may also probe further into other members of the Solanaceae family, which may reveal characteristics of the extract not previously seen in $S$. tuberosum that may prove helpful in improving its preparation.
Third, applied researches investigating other clinical applications of the extract related to its antifungal property while considering the appropriate routes of administration will be most helpful in advancing the study's practical use and formulation.

\section{Statement of Authorship}

All authors have approved the final version submitted.

\section{Author Disclosure}

All authors declared no conflicts of interest.

\section{Funding Source}

This paper was partially funded by the University of the Philippines College of Medicine - Department of Pharmacology and Toxicology, UP Medical Foundation Inc., and University of the Philippines Manila - National Institute of Health.

\section{REFERENCES}

1. World Health Organization. Prevention of hospital-acquired infections: A practical guide 2nd ed [Online] 2002 [cited 2014 October 6]. Available from http://www.who.int/csr/resources/ publications/drugresist/en/whocdscsreph200212.pdf?ua=1.

2. Batac MCR, Denning D. Serious fungal infections in the Philippines. Eur J Clin Microbiol Infect Dis. 2017; 36(6):937-41.

3. Gabrani R, Jain R, Sharma A, Sarethy IP, Dang S, Gupta S. Antiproliferative effect of Solanum nigrum on human leukemic cell lines. Indian J Pharm Sci. 2012 Sep;74(5):451-3. doi: 10.4103/0250474X.108421.

4. Friedman M. Potato glycoalkaloids and metabolites: Roles in the plant and in the diet. J Agric Food Chem. 2006;54(23):8655-81.

5. Bushway R, Bureau J, Stickney M. A new efficient method for extracting glycoalkaloids from dehydrated potatoes. J Agric Food Chem. 1985;33(1): 45-46.

6. Arendrup M, Cuenca-Estrella M, Lass-Florl C. Method for the determination of broth dilution minimum inhibitory concentration of antifungal agents for yeasts. EUCAST EDef 7.2 Revision 2012.

7. Arendrup M, Cuenca-Estrella M, Lass-Florl C. Method for the determination of broth dilution minimum inhibitory concentrations of antifungal agents for conidia forming moulds EUCAST EDef9.2 2014.

8. Ahearn D. Yeasts pathogenic for humans. The yeasts, a taxonomic study. Elsevier, Amsterdam. 1998:9-12.

9. Beighton D, Ludford R, Clark DT, et al. Use of CHROMagar Candida medium for isolation of yeasts from dental samples. J Clin Microbiol. 1995 Nov;33(11):3025-7.

10. Budtz-Jörgensen E. Etiology, pathogenesis, therapy, and prophylaxis of oral yeast infections. ActaOdontologica. 1990;48(1):61-9.

11. Budtz-Jörgensen E. Histopathology, immunology, and serology of oral yeast infections: diagnosis of oral candidosis. ActaOdontologica. 1990;48(1):37-43.

12. Manolakaki D, Velmahos G, Kourkoumpetis T, Chang Y, Alam HB, De Moya MM, et al. Candida infection and colonization among trauma patients. Virulence. 2010;1(5):367-75.

13. Kourkoumpetis TK, Velmahos GC, Ziakas PD, Tampakakis E, Manolakaki D, Coleman JJ, et al. The effect of cumulative length of hospital stay on the antifungal resistance of Candida strains isolated from critically ill surgical patients. Mycopathologia 2011;171(2):85-91.

14. Handog EB, Dayrit JF. Mycology in the Philippines. Jpn J Med Mycol. 2005(46):71-6.

15. Fidel PL, Vazquez JA, Sobel JD. Candida glabrata: review of epidemiology, pathogenesis, and clinical disease with comparison to C. albicans. Clin Microbiol Rev. 1999 Jan;12(1):80-96. 
16. Hidalgo JA, Vasquez JA. Candidiasis Treatment \& Management. [Online] 2014 [cited 2014 October 12]. Available from: http:// emedicine.medscape.com/article/213853-treatment.

17. Latgé JP. Aspergillus fumigatus and aspergillosis. Clin Microbiol Rev. 1999;12(2):310-50.

18. Hedayati M, Pasqualotto A, Warn P, Bowyer P, Denning D. Aspergillusflavus: human pathogen, allergen and mycotoxin producer. Microbiology 2007;153(6):1677-92.

19. Person AK, Chudgar SM, Norton BL, Tong BC, Stout JE. Aspergillusniger: an unusual cause of invasive pulmonary aspergillosis. J Med Microbiol. 2010 Jul;59(Pt 7):834-8. doi: 10.1099/ jmm.0.018309-0. Epub 2010 Mar 18.

20. Kaushik D, Jogpal V, Kaushik P, et al. Evaluation of activities of Solanumnigrum fruit extract. Archives of Applied Science Research 2009;1(1):43-50.

21. Sridhar T, Josthna P, Naidu C. In vitro antibacterial activity and phytochemical analysis of Solanumnigrum (Linn.)-An important antiulcer medicinal plant. Journal of Experimental Sciences 2011;2(8).

22. Karrunakaran C, Venkatesan D. Antimicrobial Activity of Selected Indian Medicinal Plants. Journal of Phytology 2010;2(2).

23. Cantelli BA, Barbosa FR, Bitencourt TA, Miranda MA, Bastos JK, Marins M, et al. Evaluation of antifungal activity of glycoalkaloids from the Solanumlycocarpum St. Hil (lobeira) in the cell membrane of dermatophyte of Trichophytonrubrum. BMC Proceedings 2014, 8(Suppl 4):P11.
24. D'Mello JF. Handbook of plant and fungal toxicants: CRC Press; 1997. pp: 19-24.

25. The Potato's Poisonous Roots [Online] 2007 [cited 2012 October]. Available from: http://www.uwm.edu/ mroffers/glycoalkaloid.htm.

26. Zhao X, Gao L, Wang J, Xu W, Hongtao B, Zhou Y. Antifungal activity of five solanaceous glycoalkaloids and their mixtures against phytopathogenic fungi Cercosporellabrassicae and Alternariaporri. Natural Product Research \& Development 2009;21(1):36.

27. Fewell AM, Roddick JG. Potato glycoalkaloid impairment of fungal development. Mycological Research 1997;101(05):597-603.

28. Rodriguez-Tudela M, Donnelly J, Arendrup M, et al. EUCAST technical note on fluconazole. Clin Microbiol Infect. 2008 Feb;14(2):193-5. Epub 2007 Dec 5.

29. Arendrup M, Rodriguez-Tudela M, Lass-Florl C, et al. EUCAST technical note on amphotericin B. Clin Microbiol Infect. 2011 Dec;17(12):E27-9. doi: 10.1111/j.1469-0691.2011.03644.x. Epub 2011 Oct 19.

30. Arendrup M, Cuenca-Estrella M, Lass-Florl C, et al. EUCAST technical notes on Aspergillus and amphotericin B, itraconazole, and posaconazole. Clin Microbiol Infect. 2012 Jul;18(7):E248-50. doi: 10.1111/j.1469-0691.2012.03890.x. Epub 2012 Apr 30. 